Eye (1990) 4, 732-736

\title{
The Value of Laboratory Testing in Uveitis
}

\author{
A. KIJLSTRA \\ Amsterdam, The Netherlands
}

\begin{abstract}
Summary
Accurate diagnosis of uveitis is of great importance since the treatment for the various uveitis entities may differ considerably. In a large number of cases the clinical picture is sufficient to make an adequate diagnosis. There are cases in which the diagnosis cannot be made on clinical grounds alone and support is needed from laboratory tests. Only a limited number of tests have been proven to be useful as a diagnostic or prognostic aid. These include HLA-B27 typing in patients presenting with anterior uveitis and testing for angiotensin converting enzyme and lysozyme in case of suspected sarcoid uveitis. Toxoplasma serology is only useful to exclude the diagnosis and a positive test has very low specific value.

Analysis of local intraocular antibody production is a valuable tool to confirm a suspected clinical diagnosis in uveitis. It is now possible to analyse paired serum and aqueous samples for the presence of specific antibodies against toxoplasma, cytomegalovirus, herpes simplex virus and varicella zoster virus using commercially available kits.

Of the patients retrospectively diagnosed as having toxoplasma chorioretinitis $\mathbf{7 5 \%}$ were shown to have a positive antibody coefficient indicating specific intraocular antibody production. Local antibody production in the eye directed against CMV confirmed the suspected diagnosis of CMV retinitis in $50 \%$ of the AIDS patients investigated. Until now we have not been able to measure local antibody production against herpes simplex virus ( 26 samples tested). Two of three patients with acute retinal necrosis had a positive antibody coefficient against varicella zoster virus. Both of these patients even had a higher titre in the aqueous than in serum. Since the choice of treatment, in infectious uveitis, depends on the causative organisms, it is very important to confirm a suspected clinical diagnosis with aqueous humor analysis.
\end{abstract}

Uveitis can be caused by infectious as well as non-infectious mechanisms. Some uveitis entities are confined to the eye, whereas others may be a symptom of systemic diseases such as ankylosing spondylitis, sarcoidosis, Behçet's disease, diabetes mellitus, multiple sclerosis or juvenile rheumatoid arthritis. Uveitis may also be caused by protozoal, bacterial, fungal, viral or helminthic infections.
The accurate diagnosis is of extreme importance because the therapy for the uveitis entities may differ markedly.

Because uveitis can be caused by so many different mechanisms, it is often extremely difficult to decide which laboratory tests should be ordered. Since severe uveitis may lead to irreversible visual loss, the practitioner is tempted to obtain as much infor-

From The Netherlands Ophthalmic Research Institute and The Dept. of Ophthalmology, University of Amsterdam.

Correspondence: Prof. Dr. A. Kijlstra, Dept. Ophthalmo-Immunology, The Netherlands Ophthalmic Research Institute, P.O. Box 12141, 1100 AC Amsterdam, The Netherlands. 
mation as possible. A non-selective ordering of tests may, however, be expensive and recent experience has shown that only a limited number of tests are really useful. ${ }^{1}$

Examination of various parameters in a peripheral bloodsample often only gives little information about isolated processes going on in the eye itself. Analysis of tissue biopsies or fluid samples from the inflamed eye could provide more insight into the disease process. In this paper I will describe the value of various tests which can currently be ordered and evaluate their value for the clinician.

\section{Uveitis entities}

Uveitis entities seen in a uveitis clinic are, of course, dependent upon regional differences (Table I). In the Netherlands the HLA-B27 associated acute anterior uveitis is the major uveitis entity ${ }^{2}$ and encompasses almost $20 \%$ of all patients seen. We consider HLA-B27 testing in patients with anterior uveitis important for an early diagnosis of ankylosing spondylitis. This test is ordered in each patient presenting with a violent anterior uveitis. No other tests specific for this patient group are routinely performed.

In patients suspected to have sarcoid uveitis two tests can be ordered. One is the angiotensin converting enzyme (ACE) level in serum

Table I Major uveitis entities in the Netherlands

\begin{tabular}{lcc}
\hline Entity & Number & \% of total \\
\hline HLA-B27 ${ }^{+}$AAU & 229 & 18 \\
Toxoplasma & 97 & 7 \\
Sarcoïd & 63 & 5 \\
Fuch's heter. cycl. & 51 & 4 \\
Pars planitis & 55 & 4 \\
Viral & 32 & 2. \\
e.c.i. & 578 & 44 \\
\hline
\end{tabular}

Rare uveitis entities in the Netherlands

\begin{tabular}{lrc}
\hline Entity & Number & \% of total \\
\hline Behçet & 10 & 0.8 \\
Birdshot & 21 & 1.6 \\
Harada & 1 & - \\
Reiter & 14 & 1.1 \\
JCA & 6 & 0.5 \\
Syphilis & 9 & 0.7 \\
Tuberculosis & 23 & 1.8 \\
Symp. Ophth. & 7 & 0.5 \\
\hline
\end{tabular}

and the other is the lysozyme serum level. ${ }^{3}$ Approximately half of the patients with sarcoid uveitis will have raised ACE and/or lysozyme levels. There are a number of points which should be considered when interpreting the tests results. First of all one should take the age of the patient into account. ACE levels are often raised in younger individuals (below 21 years old). On the other hand lysozyme levels may be increased in older patients (above 60 years old). Lysozyme levels may also be increased in patients with malfunctioning kidneys. Both ACE and lysozyme levels tend to drop after systemic corticosteroid therapy is started. This means that ordering of these tests should preferably be done before immunosuppressive therapy is begun. Advantage of this fact can be taken by repeating angiotensin converting enzyme tests to monitor the effect of therapy.

Toxoplasma chorioretinitis is one of the leading causes of posterior uveitis in the Netherlands. It is considered a late manifestation of a congenital infection. The fact that the parasite resides in the retina during the development of the embryo and can remain there for years without causing problems to the visual system is an important fact which should be considered when interpreting serological examinations. The diagnosis of ocular toxoplasmosis is based on the characteristic clinical picture of a focal necrotising retinitis. The clinical diagnosis can be confirmed by detecting toxoplasma antibodies in the blood of these cases. In a number of patients the clinical picture may be atypical or funduscopy may not be possible due to the inflammatory response in the vitreous. Various other causes should then be considered in the differential diagnosis. The presence of toxoplasma antibodies in a peripheral bloodsample is however not of great importance in view of the fact that many healthy individuals also have these antibodies resulting in a low specific diagnostic value. ${ }^{4}$ The absence of circulating antibodies on the other hand, makes the diagnosis of ocular toxoplasmosis unlikely. One problem often encountered is the fact that most parasitology laboratories are oriented towards detecting acquired toxoplasmosis infections and often deal with very high titres against the organism. For the uvei- 
tis specialist toxoplasma serology should give the answer to the question of whether the patient has ever been infected with toxoplasma. In most cases the clinical picture is so characteristic that additional testing is not necessary. In cases where the ocular symptoms are not so clear one should first begin by ordering toxoplasma serology. If this is negative one should exclude toxoplasmosis as a causal agent. If the test is positive it is compatible with the diagnosis of ocular toxoplasmosis but the disease may still be due to various other causes. In these cases additional proof may be obtained by analysing an aqueous humour sample for the presence of locally produced toxoplasma antibodies. This will be discussed in more detail later.

There are a number of tests which can be ordered using a peripheral bloodsample. These include determination of toxocara antibody levels in young children with uveitis and the measurement of antinuclear antibodies in children suspected of having uveitis associated with juvenile chronic arthritis. Furthermore, it is still routine to perform syphilis serology in all uveitis patients. A differential bloodcount and an erythrocyte sedimentation rate are also performed in all patients.

Although uveitis is considered an autoimmune disease by many and in view of the fact that well defined animal models are available showing pathogenesis of uveitis due to autoimmunity against well defined retinal antigens, the role of autoimmunity against retinal proteins in uveitis is still a matter of controversy. ${ }^{5}$ Routine testing of cellular immune reactions against various ocular proteins has not been shown to be of diagnostic value. Even for sympathetic ophthalmia, which is considered a genuine autoimmune disease of the eye, no specific laboratory test is available.

In Behçet's disease also no diagnostic laboratory tests are available. Although HLA-B5 is strongly associated with Behçet's disease in Japan and Turkey, this test is not included in the criteria for the diagnosis of this disease. Antibodies against mucosal antigens have been shown in these patients but these tests have not been developed in such a way that they are now of diagnostic use. ${ }^{6}$

Another rare entity seen in a uveitis clinic is birdshot chorioretinopathy. The diagnosis is mainly based on the clinical findings. HLA typing can be useful in these patients since $95 \%$ are HLA-A29 positive whereas the frequency in the general caucasian population is only $7 \%$.

\section{Aqueous humour analysis}

Approximately $60 \%$ of uveitis patients can be classified and the rest remain undiagnosed. Of the recognised uveitis entities the majority remains obscure as to the pathogenic mechanisms involved. Until now analysis of various immunological parameters in peripheral blood samples has not provided any clarification of this issue. A rational procedure would therefore involve the study of the inflamed tissue or the fluids bathing it. Paracentesis of the anterior chamber may provide valuable information when it is correctly aspirated and compared with parameters in a paired blood sample. It is especially useful in uveitis entities where an infectious agent is implicated.

Most experience concerning anterior chamber paracentesis to collect aqueous humour for diagnostic purposes was obtained in cases with a suspect toxoplasma chorioretinitis. ${ }^{7}$ Of the patients $(n=24)$ who were finally diagnosed as having a toxoplasma chorioretinitis, $75 \%$ had a positive coefficient. ${ }^{8}$

A negative coefficient was found in five cases. In three of these cases no antibody was detectable in the aqueous. In two other cases we did detect specific antibody in the aqueous but could not rule out that this was due to leakage from the blood compartment.

Local antibody production in the eye directed against CMV confirmed the suspected diagnosis CMV retinitis in $50 \%$ of AIDS patients $(n=16)$ investigated. ${ }^{9}$ In Table II some examples are shown of the analysis of CMV antibodies in the aqueous humour obtained from AIDS patients with a suspected CMV retinitis. Serum IgG levels are often markedly raised in these patients and values as high as $36 \mathrm{mg} / \mathrm{ml}$ can be observed. IgG levels in aqueous are comparable to those seen in toxoplasma patients (data not shown).

Testing for intraocular production against herpes simplex virus was negative in all samples $(n=26)$ tested until now, including three patients with acute retinal necrosis. 
Table II Intraocular CMV antibody synthesis in patients with AIDS and retinitis

\begin{tabular}{|c|c|c|c|c|c|}
\hline Patient & Serum titre & Aqueous titre & Serum IgG & Aqueous IgG & $\mathrm{C}$ \\
\hline 1 & 128 & 8 & 18.1 & $>1.0$ & $<1$ \\
\hline 2 & 5120 & 8 & 25.1 & 0.042 & 0.9 \\
\hline 3 & $<2$ & NEG & 17.6 & 0.1 & - \\
\hline 4 & 80 & 8 & 18.0 & 0.2 & 9.0 \\
\hline 5 & 16000 & 32 & 25.2 & 0.024 & 2.1 \\
\hline 6 & 100 & 2 & 24.2 & 0.028 & 17.3 \\
\hline 7 & 40 & NEG & 15.9 & 0.14 & - \\
\hline 8 & 20 & 4 & 25.3 & 0.05 & 101 \\
\hline 9 & 400 & 8 & 17.3 & 0.128 & 2.7 \\
\hline 10 & 400 & NEG & 15.7 & 0.07 & - \\
\hline 11 & 2000 & 128 & 15.4 & 0.06 & 16.4 \\
\hline 12 & 320 & 8 & 36.8 & 0.11 & 8.4 \\
\hline 13 & 200 & 2 & 22.7 & 0.01 & 23 \\
\hline 14 & 640 & 16 & 20.4 & 0.078 & 6.5 \\
\hline 15 & 32 & NEG & 15.5 & 0.024 & - \\
\hline 16 & 64 & 2 & 13.0 & 0.088 & 4.6 \\
\hline
\end{tabular}

The titres represent the highest serum or aqueous dilution still giving a positive test result. The IgG levels are in $\mathrm{mg} / \mathrm{ml}$. The $\mathrm{C}$ value represents the antibody coefficient. A coefficient above 3.0 is arbitrarily considered positive.

Two of the three patients with acute retinal necrosis had a positive antibody coefficient against varicella zoster virus. Both of these patients even had a higher titre in the aqueous than in serum.

\section{Discussion}

Only a limited number of relevant laboratory tests are currently available to the ophthalmologist dealing with uveitis patients. The battery of tests often performed in the past have often not given satisfactory answers. In cases with severe uveitis where both clinical judgement and traditional laboratory testing have failed to give an answer we would advocate performing an anterior chamber paracentesis and a tailored analysis of the aqueous humour in combination with a paired serum sample. It should be stressed that an isolated analysis of aqueous is worthless since the presence of antibodies or antigens in the aqueous may be due to blood aqueous barrier breakdown.

Determination of specific antibody production in the eye is a valuable diagnostic aid in the workup of uveitis patients. It represents an indirect proof of the role of a microorganism in the pathogenesis of the intraocular inflammation. Direct proof would involve the culture of the microorganism or the detection of its antigens. The culture of aqueous samples in uveitis has not been very helpful.
In the future techniques will become available to detect the free antigens of a suspected microorganism or its complex with antibody.

Interpretation of test results is not always an easy task. Negative test results can be due to analysis of aqueous samples during an episode of low inflammatory activity or may be due to over-correction caused by leakage of plasma proteins into the aqueous. It is not yet clear where the antibody synthesis occurs in infectious uveitis entities and how intraocular antibody reaches the aqueous humour. In this context it is of interest that we have recently found very high toxoplasma antibody titres in a vitreous sample of a suspected toxoplasma chorioretinitis patient which exceeded the serum titre by four-fold. Although this possibly represents an incidental case it may point to local antibody .concentration differences within the eye.

Determination of local antibody production is relevant for the initial diagnosis and the subsequent choice of therapeutic measures to be taken. We have also observed that it may be useful in monitoring the effect of therapy in a small number of cases. ${ }^{9}$ Analysis of cells in an aqueous or vitreous sample may be important for the diagnosis of masquerade syndromes (intraocular tumours). Analysis of ocular fluid samples therefore requires close collaboration between specialised ophthalmo-immunology, microbiology and pathology laboratories. 
This diagnostic approach is now being used by several centres in Europe and Japan but is not generally performed in the United States nor the United Kingdom. Until now no complications have been reported after aqueous chamber paracentesis. In certain unknown uveitis entities aqueous humour analysis may identify the causal organisms and be helpful in precisely identifying the clinical picture. In future cases with similar typical clinical findings aqueous analysis will therefore not always be needed. A stepwise approach may also be considered; first testing the serum for antibodies. If a negative result is obtained it is unlikely that the patients will have the suspected uveitic disease. If positive one may proceed and collect a paired sample of aqueous and serum.

False positive results have been reported by Dussaix et al. ${ }^{10}$ To eliminate this, local antibody production against at least two different agents is tested. A high coefficient against different antigens at the same time may raise suspicion concerning the test results or the uveitic condition of the patient. To date no aqueous samples have been encountered in our laboratory with a positive coefficient for more than one infectious agent.

It is likely that the amount of different organisms (Candida, Borrelia etc.) to which local ocular antibody production can be detected will increase in the near future. Since the choice of therapy, especially in infectious uveitis entities, depends directly on the causative organism, it is of utmost importance to confirm a clinical suspicion with a tailored ordering of aqueous humour tests. It can be expected that the classical immunological tests discussed above will soon be replaced by modern DNA techniques, employing the polymerase chain reaction to amplify genetic material of suspected microorganisms residing in the eye.

\section{References}

${ }^{1}$ Kijlstra A, Linssen A, Ockhuizen Th, Baarsma GS, Dandrieu MR, Tjoa St, de Graauw J, Christiaans BJ, de Jong PTVM, Dekker-Saeys AJ, Feltkamp TWE: Diagnostic tests in uveitis. In Saari KM ed. Uveitis Update. Amsterdam, Elsevier Sci Publ B.V. 1984: 421-25.

${ }^{2}$ Kijstra A, Rothova A, Baarsma GS, Zaal MJM, Fortuin ME, Schweitzer CMC, Glasius E, de Jong PTVM: Computer registration of uveitis patients. Doc Ophthalmol 1987, 67: 139-43.

${ }^{3}$ Baarsma GS, La Heij E, Glasius E, de Vries J, Kijlstra A: The predictive value of serum angiotensin converting enzyme and lysozyme levels in the diagnosis of ocular sarcoidosis. Am J Ophthalmol 1987, 104: 211-7.

${ }^{4}$ Rothova A, van Knapen F, Baarsma GS, Kruit PJ, Loewer-Sieger DH, Kijlstra A: Serology in ocular toxoplasmosis. Br J Ophthalmol 1986, 70: 615-22.

${ }^{5}$ Kijlstra A, Hoekzema R, Van der Lelij A, Doekes G, Rothova A: Humoral and cellular immune reactions against retinal antigens in clinical disease. Curr Eye Res 1990, 9 (Suppl): 85-9.

${ }^{6}$ Klok AM, de Vries J, Rothova A, Zaal MJM, Schweitzer CMC, Bos JD, Kijlstra A: Antibodies against ocular and oral antigens in Behçet's disease associated with uveitis. Curr Eye Res 1989, 8: 957-62.

${ }^{7}$ Kijlstra A, Luyendijk L, Baarsma GS, Rothova A, Schweitzer CMC, Timmerman Z, de Vries J, Breebaart AC: Aqueous humor analysis as a diagnostic tool in toxoplasma uveitis. Int Ophthalmol 1989, 13: 383-6.

${ }^{8}$ Kijlstra A, van den Horn GJ, Luyendijk L, Baarsma GS, Schweitzer CMC, Zaal MJM, Timmerman Z, Beintema M, Rothova A: Laboratory tests in uveitis: new developments in the analysis of local antibody production. Doc Ophthalmol 1990, (in press).

${ }^{9}$ Luyendijk L, van der Horn GJ, Visser OHE, Suttorp-Schulten MSA, van de Biesen PR, Rothova A, Kijlstra A: Detection of locally produced antibodies to herpes viruses in aqueous of patients with acquired immune deficiency syndrome (AIDS) or acute retinal necrosis syndrome (ARN). Curr Eye Res 1990, 9 (Suppl): 7-11.

${ }^{10}$ Dussaix E, Cerqueti PM, Pontet F, Bloch-Michel E: New approaches to the detection of locally produced antiviral antibodies in the aqueous of patients with endogenous uveitis. Ophthalmologica 1987, 194: 145-9. 